\title{
Favre- and Reynolds-averaged velocity measurements: interpreting PIV and LDA measurements in combustion
}

M. Mustafa Kamal, Saravanan Balusamy, Ruigang Zhou, Simone Hochgreb

Department of Engineering, University of Cambridge, CB2 1PZ, United Kingdom

Corresponding author: M. Mustafa Kamal

Department of Engineering, University of Cambridge

Cambridge, CB2 1PZ, United Kingdom

Fax: (+44) (0) 1223764311

Email:mmk44@cam.ac.uk

Color reproduction: no (color line figures are to be printed in gray scale) 


\title{
Favre- and Reynolds-averaged velocity measurements:
}

\section{interpreting PIV and LDA measurements in combustion}

\author{
M. Mustafa Kamal*, Ruigang Zhou, Saravanan Balusamy, Simone Hochgreb \\ Department of Engineering, University of Cambridge, CB2 1PZ, UK
}

\begin{abstract}
Previous studies using particle image velocimetry (PIV) and laser Doppler anemometry (LDA) have raised the question of how these measurements should be compared. This study reports on the difference between Favre-averaged and Reynolds-averaged velocity statistics for a turbulent burner using PIV and LDA for unconditional and conditional velocity measurements. The experimental characterization of flow fields of premixed and stratified methane/air flames is carried out under globally turbulent lean conditions (global equivalence ratio at 0.75$)$, over a range of stratifications and swirl numbers. Unconditioned velocity data was acquired using aluminium oxide to seed the flow field. Conditioned measurements were performed using vegetable oil aerosol as seed, which burns through the flame front, thus allowing only the non-reacting flow velocities to be obtained. A critical comparison of unconditioned velocity profiles measured using both PIV and LDA, including axial, radial, and tangential components is made against conditioned and reconstructed mean velocities at different cross-sections of the flame. The comparison reveals how the differences between the Favre-averaged (unconditioned) and the Reynolds-averaged (conditioned) velocity measurements in the flame brush region can be accounted for using the mean progress of reaction, and highlights the limits of the accuracy and agreement between PIV and LDA measurements.
\end{abstract}

*Corresponding author. E-mail: mmk44@cam.ac.uk. Tel: +44 (0)1223764098. 
Keywords: Turbulent flame; premixed flame; LDA; PIV; Favre averaging 


\section{Introduction}

Recent progress towards the understanding of premixed turbulent combustion and validation of numerical simulation models has relied on detailed databases for a variety of scalar and velocity measurements in premixed and stratified flames [1] [2] [3] [4] [5]. Recent work by Zhou et al. [4] directly compared low speed measurements of velocities and their statistical moments in a stratified swirl burner using laser Doppler anemometry (LDA) and low speed particle image velocimetry (PIV). Unlike prior work (Nomura et al. [6] and Seffrin et al. [1]), both the velocities, their moments and the integral length scales measured with both techniques were in excellent agreement, as shown in Fig. 1. Although such concordance is generally considered a success, the agreement between measurements was puzzling: as explained further on, LDA relies on local time-based bursts of particle passage, in proportion to the particle (and gas) density, whereas PIV measurements are number averaged images. Therefore, LDA measurements have in general been considered Favre (density) averaged [7] [8], whilst PIV measurements should be Reynolds (number) averaged. There should therefore be an observable difference within the flame brush. We have therefore undertaken a set of experiments using aerosols to generate velocity measurements in the unreacted zone only, in order to clarify the differences and understand under what conditions PIV and LDA measurements should agree.

In the following sections, we describe the measurement principles and how they were executed, the comparison between LDA and PIV conditioned and unconditioned measurements, and connect the various measurements using what would be expected using a thin flame model.

1.1 Velocity measurements

Measurements of velocity in flames rely chiefly on optical methods such as LDA and 
PIV. In both cases, sufficiently small particles or aerosols are used to seed the mixture, which can follow the relevant turbulent frequencies. In LDA, velocities are determined from the signal produced by Mie scatter of a particle travelling through a millimetre-long laser light fringe volume. The frequency of the bursts is therefore proportional to the number of particles crossing the probe volume per unit time, and thus to the density of the gas and the velocity of the particles. If the particle seeding is uniform, the probability of crossing is inversely proportional to the velocity, thus LDA measurements are routinely corrected for the velocity bias by the transit time between particles [9] [10]. However, the expansion of gases in flames means that in the region of the flame brush, the particle density is proportional to the gas density, so that the measurements will be weighted accordingly, in what is called density or Favre weighting.

In PIV, a twin set of images of the Mie scatter is taken spaced by time, and the displacements and velocities are obtained via spatial correlation of the two images. Measurements in a particular region are averaged over a spatial interrogation window, typically of the order of 16 to 32 pixels square, often corresponding to sub-millimetre resolutions. Ensembles of measurements are averaged, yielding an equally weighted measurement for each sample set of images, in what is called number or Reynolds averaging.

Most common flames are thin relative to the overall domain, with typical thicknesses under $0.5 \mathrm{~mm}$ at atmospheric conditions. The flame thickness is comparable or slightly smaller than the spatial resolution of LDA and PIV. Therefore, most measurements are made either in the reactant or product zones, with very low probability of measurements within the flame zone itself. Measurements within the narrow flame zone would be automatically weighted by density for interrogation windows of the same order of magnitude as the thickness of the flame, thus averaging velocities according to the number of particle and autocorrelation pairs in the image set. We can therefore say that for measurements around the 
flame brush of turbulent flames, we expect PIV measurements to be Reynolds averaged and LDA measurements to be Favre averaged. Under what conditions would the averages of velocity components be similar or identical? We address this question in the following section.

\subsection{Averaging in the thin flame limit}

In the thin flame or BML model [11] [12], we have a description of the flame in two zones for reactant and product, corresponding to a progress variable $c$, where $\mathrm{c}=0$ in the reactants and unity in the products. The local probability of $c$ is denoted $p(c)$, and the corresponding average or density averaged values of a scalar $\bar{\psi}$ or $\tilde{\psi}$ can be correspondingly calculated as $\quad \bar{\psi}=\int_{0}^{1} \psi(c) p(c) d c=(1-\bar{c}) \psi_{r}+\bar{c} \psi_{p} \quad, \quad$ and $\tilde{\psi}=1 / \bar{\rho} \int_{0}^{1} \psi(c) \rho(c) p(c) d c=(1-\bar{c}) \psi_{u}+\bar{c} \psi_{b}$, where the subscripts $r$ or $p$ indicate reactant and product, $\rho$ is the density, and the mean density is $\bar{\rho}=\int_{0}^{1} \rho(c) \psi(c) p(c) d c=$ $(1-\bar{c}) \rho_{r} \psi_{r}+\bar{c} \rho_{p} \psi_{p}$. Following the original model, we use a temperature rise ratio $\tau=\left(T_{p}-T_{r}\right) / T_{r}$ to quantify the density difference. The Favre-averaged progress of reaction thus follows as $\tilde{c}=\bar{c} /((\tau+1)-\tau \bar{c})$. Now we are ready to express the Favre averaged velocity components $\tilde{u}_{i}$ as a function of the Reynolds average values of the properties in the reactant and product regions, and the mean progress of reaction as:

$$
\begin{aligned}
& \tilde{u}=(1-\tilde{c}) \bar{u}_{r}+\tilde{c} \bar{u}_{p} \\
& \bar{u}=(1-\bar{c}) \bar{u}_{r}+\bar{c} \bar{u}_{p}
\end{aligned}
$$

Subtracting Eq. (1b) from (1a), we have:

$$
\bar{u}-\tilde{u}=(\bar{c}-\tilde{c})\left(\bar{u}_{p}-\bar{u}_{r}\right)
$$

We therefore have the result that the difference between the Favre and Reynolds average velocities should be proportional to the difference in local velocities of the reactants 
and products, weighted by the difference between Reynolds and Favre averaged progress of reaction. The latter term can be determined from the definition of the progress of reacting in the limit of a thin flame as:

$$
(\bar{c}-\tilde{c})=\tau c(1-\bar{c}) /(\tau(1-\bar{c})+1)
$$

Figure 2 shows that the difference peaks at a value of around 0.4 at a progress of reaction approximately 0.7 towards the product zone for a range of values of $\tau$ compatible with the present experiments and flames in general. This means that even if there are significant velocity differences between reactants and products, the difference between Favre and Reynolds averaged velocities should be smaller than 0.4 times the respective value, particularly at the edges of the flame brush.

In what follows, we test the hypothesis above by comparing measurements obtained within a flame brush using particle-seeded flows with LDA and PIV to obtained the unconditioned velocity components $\tilde{u}_{i}$ and $\bar{u}_{i}$, and aerosol seeded flows using PIV to obtain the conditioned reactant velocity $\tilde{u}_{r i}=\bar{u}_{r i}$. This allows us to obtain $\tilde{u}_{p i}$ from equation (1b) and the right and left hand sides of equation (2) for comparison, if the local pdf of progress of reaction is available.

\section{Experimental setup and methods}

\subsection{Cambridge/Sandia Stratified Swirl Burner (SwB)}

The SwB burner was designed to generate reacting flow conditions representative of turbulent flows in practical systems, including sufficiently high turbulence levels, swirl, and operation under premixed and stratified conditions. The swirl burner geometry described in [3] [13], and a cross section and top view are shown in Figure 3. 
The burner consists of co-annular tubes with a development length exceeding 25 hydraulic diameters to ensure well developed turbulent flow. A ceramic central bluff body is used to stabilize the flame with minimal heat loss. Mass flow controllers are used to control the inner annulus equivalence ratio $\left(\phi_{i}\right)$ and the outer equivalence ratio $\left(\phi_{o}\right)$ independently, allowing the stratification ratio $\left(S R=\phi_{i} / \phi_{o}\right)$ to be easily varied, for a fixed global stratification ratio $\left(\phi_{g}\right)$. In the present paper we do not consider the effects of swirl.

\subsection{Operating Conditions}

The operating conditions for the present study are shown in Table 1 . The generalized notation $S w B N_{z}$ is used to denote the test cases, where $N$ is the case number and $z$ is the downstream distance in millimetres. The bulk velocity in the outer annulus, $U_{o}=18.7 \mathrm{~m} / \mathrm{s}$, was set at more than twice the value of the velocity in the inner annulus, $U_{i}=8.3 \mathrm{~m} / \mathrm{s}$, in order to generate substantial levels of shear and thus turbulence between the two flows. Coflow air was supplied around the outer annulus with a bulk velocity $U_{c o}=0.4 \mathrm{~m} / \mathrm{s}$ to provide well-characterized boundary conditions. The Reynolds numbers derived from the bulk velocities at the exit geometry are $R e_{i}=5960$ for the inner flow and $R e_{o}=11500$ for the outer flow. The stratification ratio was varied from unity for premixed cases to 3 for the most stratified cases. The inner, outer, and co-flow were each seeded with $1 \mu \mathrm{m}$ calcined aluminium oxide particles in case of unconditioned measurements, and with vegetable oil aerosol in case of conditioned measurements. The solid particle seeding was achieved by passing a portion of each air flow through simple turbulent fluidized seeders, and adjusting the fraction of the total flow through each seeder using needle valves to ensure similar seed density in inner, outer and co-flow streams. For the aerosol, a high air velocity atomizer was used to produce a monodisperse mist of aerosol droplets with a mean diameter of about $1 \mu \mathrm{m}$ as measured by an electronic low pressure impactor. The characteristic time scale for drag on 
a particle in Stokes flow is $\tau_{p}=\rho_{p} d_{p}^{2} / 18 \mu_{a}$, where $\rho_{p}$ and $d_{p}$ are the particle density and diameter, respectively, and $\mu_{a}$ is the dynamic viscosity of the surrounding fluid, the calculated time scale $\tau_{p}$ is of the order of $10^{-5}$ seconds, for this particle size, velocity fluctuation frequencies of up to tens of $\mathrm{kHz}$ can be followed.

\subsection{PIV measurements}

The PIV system consists of a double-pulsed Nd:YAG laser (Litron Nano PIV) operating at $532 \mathrm{~nm}$ and $100 \mathrm{~mJ} /$ pulse shaped into an expanding beam. The time delay between each pulse was optimised to $10 \mu$ s based on the interrogation window size and the maximum displacement of particle pairs to be within $1 / 4$ of the interrogation window size. The light scattered by the seed particles was imaged using a CCD camera (LaVision Imager Pro X 4M) fitted with a Nikon AF Micro Nikkor $60 \mathrm{~mm}$ lens (f/4) and a $50 \mathrm{~mm}$ interference filter centred about $532 \mathrm{~nm}$, at 90 degrees to the laser sheet. The $2048 \times 2048$ pixel imaged field was $60.3 \mathrm{~mm}$ wide by $60.3 \mathrm{~mm}$ tall, yielding a pixel resolution of $29.4 \mu \mathrm{m}$. The PIV system was operated at $7 \mathrm{~Hz}$ and 2000 images were recorded for each condition.

All PIV images were processed using LaVision software (Davis 7.4). For the twocomponent PIV, raw images were pre-processed by subtracting a sliding background ( 3 pixel scale length) and normalizing the particle intensity using a $\min / \max$ filter ( 3 pixel scale length) and normalizing the particle intensity using a min/max filter (3 pixel scale length). Vectors were calculated using multi-pass cross-correlation with an initial window size of 64 $\times 64$, decreasing to a $32 \times 32$ window size in the final three passes. The window overlap was held constant at $50 \%$ in each pass. This gives a vector spatial resolution of $0.47 \mathrm{~mm} / \mathrm{vector}$. All vector fields were filtered via rejection for a Q-factor (ratio of highest to second highest peaks in the displacement correlation map) below 1.2, and the resulting fields were median 
filtered. Isolated regions with fewer than five vectors were removed. No interpolation was done to for missing vectors. The full scale velocity measurement error can be determined by the ratio of the nominal correlation peak value $(0.2$ pixels for window of $12 \times 12$ pixels $)$ to the maximum displacement permitted, namely $1 / 4$ of the final interrogation window. Thus the measurement error for the two-component PIV $(32 \times 32)$ is calculated to be $\pm 1.3 \%$ of full scale.

\subsection{Pairwise LDA configuration}

The LDA used is a Dantec two-component system. The laser used is an Ar-ion continuous wave laser (Spectra-Physics Stabilite 2017) operating at $514.5 \mathrm{~nm}$ and $488 \mathrm{~nm}$ with power set to $1.5 \mathrm{~W}$. The signals generated by scattering are transferred to the burst spectrum analyser processor through a Nikon Micro Nikkor $105 \mathrm{~mm}$ lens (f/2.8), a pinhole, a Dantec colour separator and photodetectors, for measurements of two velocity components. The receiving optics collect light at an off-axis forward position at an angle of $30^{\circ}$. The calculated dimensions of the LDA probe volume are $3.40 \mathrm{~mm}$ length $\times 0.14 \mathrm{~mm}$ width $\times 0.14$ $\mathrm{mm}$ height, which yields an effective probe volume of $0.91 \times 0.14 \times 0.13 \mathrm{~mm}$ after considering the magnification of the lens (determined by focal length and focusing distance), and the angle. A pairwise LDA setup was achieved by rotating the system by 90 degrees and scanning along the same positions to obtain the third velocity component. As a result, all three velocity components are resolved at the measurement locations with at least $10 \mathrm{kHz}$ data rate. Transit time weighting is applied to minimize the velocity bias due to random sampling. Radial symmetry of the flow is verified by acquiring full radial scans. The measurements were taken at multiple axial locations, corresponding to previous scalar measurements [3] [13]. The sampling spatial resolution (spatial interval between each measurement point) is $1 \mathrm{~mm}$. The random errors introduced for the mean and RMS velocity 
measurements in LDA are $0.7 \%$ and $1.4 \%$ corresponding to the finite sample size of LDA data $(\sim 10,000$ data per measurement point). Further details of the LDA processing and optimization are available in [4].

\subsection{Progress of reaction measurements}

Measurements of progress of reaction were made by considering the Mie scatter signal of a total of 2000 images of vegetable oil droplets used in the PIV. Vegetable oil evaporates and burns at temperature above about $600 \mathrm{~K}$. The evaporation time of the aerosol droplets (15 $\mathrm{s})$ is very large compared to the time step between two laser pulses $(10 \mu \mathrm{s})$ and the time needed for a droplet to travel through the field of view $(3 \mathrm{~ms})$. The Mie signal interface marks an approximate isothermal contour (around $650 \mathrm{~K}$ ) [14] and, in the thin flame approximation, also separates products and reactants. The flame thickness is of the order of $0.2 \mathrm{~mm}$ [3], thus sufficiently thin for the present approximation. A low luminosity signal arising from the filtered flame emission marks the product region present near the interface. In order to determine the appropriate flame interface, a histogram-based thresholding method is adopted for each Mie scattered image. As signal-to-noise ratios in images vary due to beam profile inhomogeneities and variations in seeding concentration, a modified algorithm based on the work of Weiß et al. [15] is employed, such that the interface is determined as the position of the maximum gradient in the Mie scattering intensity field. A preliminary threshold calculated from the global intensity histogram, coupled with a ridge finding algorithm is implemented to detect the maximum gradient. The algorithm showed little sensitivity to the threshold value over a wide range, and the results for the progress of reactions are robust compared to measurements based on temperature [3]. The ensemble of thresholded and binarised images is averaged to construct a mean flame brush. 


\section{Results and Discussion}

\subsection{Conditioned measurements}

Figure 4 presents radial profiles of mean axial velocity for all the three cases at $\mathrm{z}=30 \mathrm{~mm}$ obtained from conditioned (aerosol only) PIV and LDA measurements. As expected, the conditioned PIV results are in excellent agreement with the conditioned LDA measurements. The mean progress of reaction obtained from the aerosol measurements is shown in Fig. 5. The flame region widens with the expansion of the flame products, and the flame brush region widens due to the action of turbulence further downstream in all cases. The effect of stratification from the premixed (SwB1) to the most stratified (SwB9) flame is to widen the flame due to higher inner temperatures and overall rate of heat release.

\subsection{Conditioned and unconditioned velocities}

Radial profiles of the mean velocity components from both the conditioned and unconditioned LDA measurements are shown for all cases in Fig. 6. The mean progress variable is also overlaid on the same figure for individual cases. The velocity profiles have been discussed in [4]: the flow leaves the annular channels as fully developed flow, and a shear layer emerges and develops. The flame stabilizes at the edge of the bluff body and develops in a projected triangular cross section emerging from the bluff body vertex, as shown in Fig. 5 and highlighted in the shaded area in Fig. 6. The conditioned and unconditioned profiles for the radial and tangential velocities are indistinguishable from the conditioned measurements within the accuracy of the present measurements. Since the nonaxial velocity component values are very low in the present non-swirling flows, they are not considered further. The axial measurements are in agreement except within the flame brush 
and product region, where they depart. The discrepancy is particularly large in the region near the burner, where the conditioned velocity at the edge of the flame brush is almost twice the value of the unconditioned velocity. Further downstream however, the axial velocity measurements of conditioned and unconditioned velocities are closer, within approximately 10-20 percent.

The conditioned and unconditioned velocities obtained from aerosol and particle seeding can be used to determine the conditioned product velocity via Eq. (1), as a function of the progress of reaction, as shown in Fig. 7. The product velocity is much lower than the reactant velocities within the flame brush. This is a result of the lower unconditioned velocity within the region. The effect is more pronounced in the cases with higher heat release rate (SwB9), and further downstream.

The calculated difference between the PIV-derived Reynolds averaged unconditioned measurements for axial velocities $\bar{u}$, and the Favre averaged unconditioned velocities $\tilde{u}$ (Fig. 1), here called left hand side, LHS (of Eq. (2)), can be plotted against the progress of reaction along with the expected difference of these two quantities from the right hand side (RHS) of Eq. (2). The RHS is the product of the difference in Favre averaged and Reynolds averaged progress of reaction (from Eq. (3) and Fig. (6)) times the difference in Reynolds averaged velocities of product and reactant from Fig. 7. Figure 8 shows the outcome: despite the significant differences between the product and reactant velocities, the difference in the Reynolds and Favre averages is indeed very small, and explains the surprising agreement between the LDA and PIV measurements.

\section{Conclusions}


We have shown the reason why PIV and LDA measurements within the flame brush may not differ significantly in the approximation of thin flames, and demonstrated the principle for a particular case, in which there are significant differences between reactant and product velocities and densities.

Unless there are very large differences in velocities in the middle region of the flame brush where the density-weighted progress of reaction is highest, the difference is minimized because it is proportional to the gap between averaged progress of reaction and its density weighted version, which is a small number. Exceptions of course must be made for situations where the thin flame model does not apply. We suggest that in general LDA and PIV measurements in flame brushes will lead to somewhat different results, and the experimenter should be aware of what conditions apply in the particular situation. The present data can be made available by contacting the authors.

\section{Acknowledgments}

The authors would like to thank the University of Engineering and Technology Peshawar (Pakistan) and the University of Cambridge for their financial contributions to this work.

\section{References}

[1] F. Seffrin; F. Fuest; D. Geyer; A. Dreizler, Combust. Flame 157 (2) (2010) 384-396

[2] G. Kuenne; F. Seffrin; F. Fuest; T. Stahler; A. Ketelheun; D. Geyer; J. Janicka; A. Dreizler, Combust. Flame 159 (8) (2012) 2669-2689

[3] M. S. Sweeney; S. Hochgreb; M. J. Dunn; R. S. Barlow, Combust. Flame 159 (2012) 2896-2911

[4] R. Zhou; S. Balusamy; M. S. Sweeney; R. S. Barlow; S. Hochgreb, Combustion and Flame 160 (10) (2013) 2017-2028

[5] M. M. Kamal; C. Duwig; S. Balusamy; R. Zhou; S. Hochgreb, in: Proceedings of the ASME Turbo Expo, GT2014-26222, Dusseldorf, Germany, accepted, 2014. 
[6] T. Nomura; Y. Takahashi; T. Ishima; T. Obokata, in: 12th International Symposium on Applications of Laser Techniques to Fluid Mechanics, 2004.

[7] R. K. Cheng, Comb. Sci. Tech. 41 (1984) 109-142

[8] S. N. Oka; V. V. Bakic, in: Engineering Turbulence Modelling and Experiments, W. Rodi; G. Bergeles, (Eds.) Elsevier: Oxford, 1996; Vol. 3, pp 807-816.

[9] W. K. George, Experimental Thermal and Fluid Science 1 (1988) 29-40

[10] C. Tropea; A. L. Yarin; J. F. Foss, Springer Handbook of Experimental Fluid Mechanics, Springer-Verlag Berlin Heidelberg, 2007.

[11] K. N. C. Bray; P. A. Libby; G. Masuy; J. B. Moss, Comb. Sci. Tech. 25 (1981) 127-141

[12] R. K. Cheng; I. G. Shephard, Comb. Sci. Tech. 49 (1986) 17-35

[13] M. S. Sweeney; S. Hochgreb; M. J. Dunn; R. S. Barlow, Combust. Flame 159 (2012) 29122929

[14] P. C. Miles; F. C. Gouldin, Proc. Combust. Inst. 24 (1992) 477-484

[15] M. Weiß; N. Zarzalis; R. Suntz, Combust. Flame 154 (4) (2008) 671-691 


\section{Figure captions}

Fig. 1. Radial profiles of mean axial velocity for unconditioned PIV and LDA, extracted at $z=30 \mathrm{~mm}$.

Fig. 2. Difference between Reynolds and Favre averaged progress of reaction values in the thin flame limit, as a function of progress of reaction and normalized flame temperature rise. The values for $\tau=3.6$ and 6.5 are based on the equilibrium temperatures associated with the minimum and maximum equivalence ratios in stratified cases, and $\tau=3.6$ refers to the equivalence ratio for the premixed case.

Fig. 3. Burner geometry. Top: top view, bottom: Exit geometry. The arrows indicate the direction of flow and swirl.

Fig. 4. Radial profiles of conditioned reactant mean axial velocity PIV and LDA, extracted at $z=30 \mathrm{~mm}$.

Fig. 5. Mean progress of reaction $\bar{c}$. Linear gray scale from black: product; white: reactant.

Fig. 6. Radial profiles of LDA measurements of mean conditioned reactant velocities $u, v, w$ representing axial, radial, and tangential directions (solid lines), the unconditioned LDA measurements (symbols), and progress of reaction (solid black line) for three different axial positions (rows) for case SwB1 (left), SwB5 (middle) and SwB9 (right).

Fig. 7. Radial profiles of calculated product and measured conditioned reactant axial velocities extracted at three different axial positions (rows) in the flame brush region (rows).

Fig. 8. LHS $=(\bar{u}-\tilde{u})$, and RHS $=(\bar{c}-\tilde{c})\left(\bar{u}_{r}-\bar{u}_{p}\right)$ from Eq. (2) as a function of progress of reaction, extracted at three different axial positions (rows) for all the three cases 
Table 1.

Operating conditions, for global $\phi_{g}=0.75$.

\begin{tabular}{cccc}
\hline Case & $\boldsymbol{\phi}_{\boldsymbol{i}}$ & $\boldsymbol{\phi}_{\boldsymbol{o}}$ & $\mathrm{SR}$ \\
\hline$S w B 1$ & 0.75 & 0.75 & 1 \\
$S w B 5$ & 1 & 0.5 & 2 \\
$S w B 9$ & 1.125 & 0.375 & 3 \\
\hline
\end{tabular}




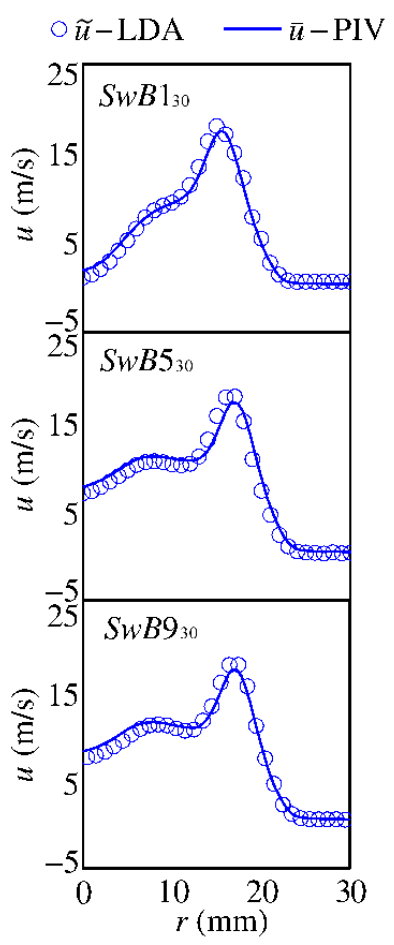

Fig. 1. Radial profiles of mean axial velocity for unconditioned PIV and LDA, extracted at $z=30 \mathrm{~mm}$. 


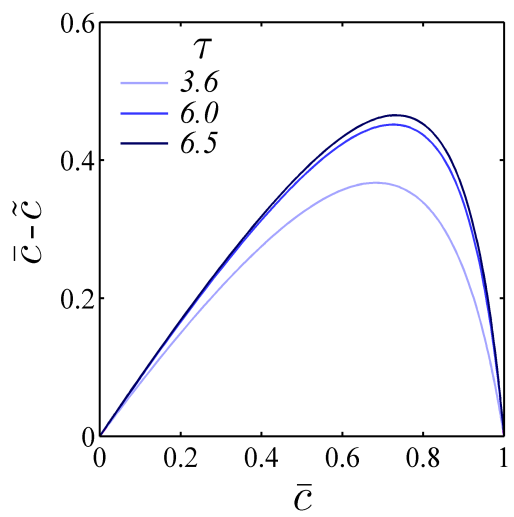

Fig. 2. Difference between Reynolds and Favre averaged progress of reaction values in the thin flame limit, as a function of progress of reaction and normalized flame temperature rise. The values for $\tau=3.6$ and 6.5 are based on the equilibrium temperatures associated with the minimum and maximum equivalence ratios in stratified cases, and $\tau=3.6$ refers to the equivalence ratio for the premixed case. 

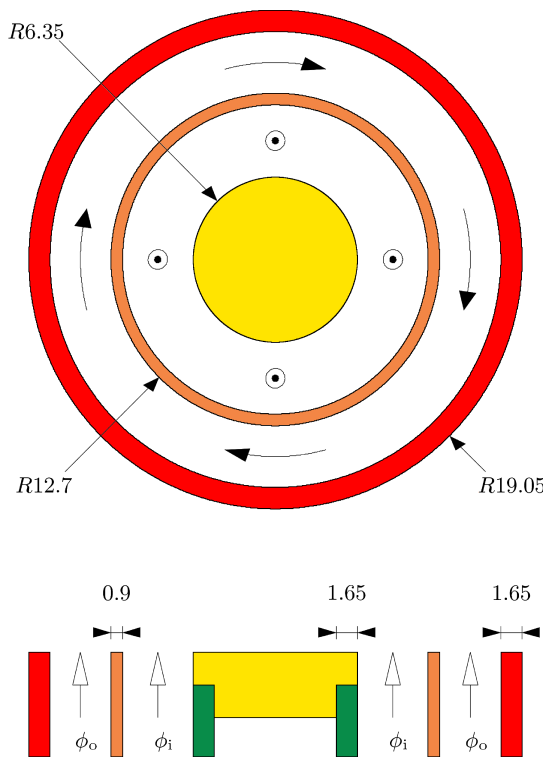

Fig. 3. Burner geometry. Top: top view, bottom: cross section. The arrows indicate the direction of flow and swirl. 


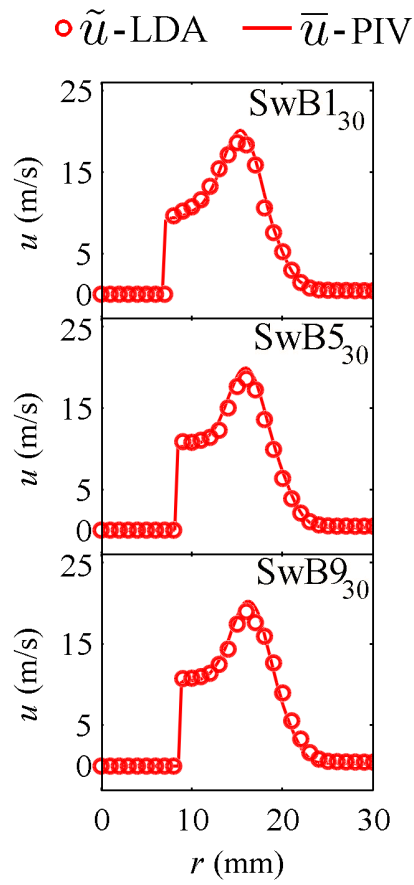

Fig. 4. Radial profiles of conditioned reactant mean axial velocity PIV and LDA, extracted at $z=30 \mathrm{~mm}$. 


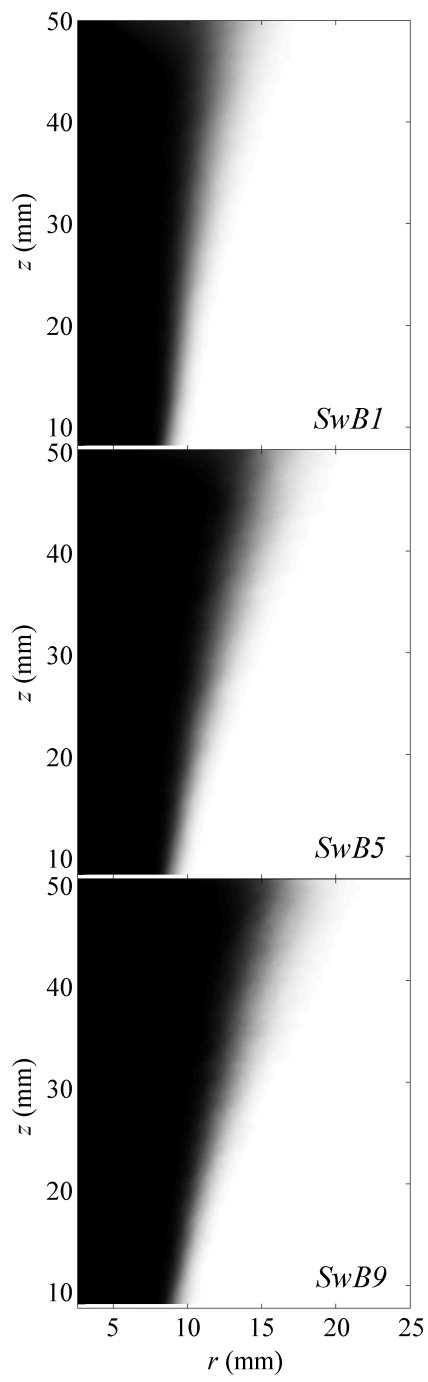

Fig. 5. Mean progress of reaction $\bar{c}$. Linear gray scale from black: product; white: reactant. 


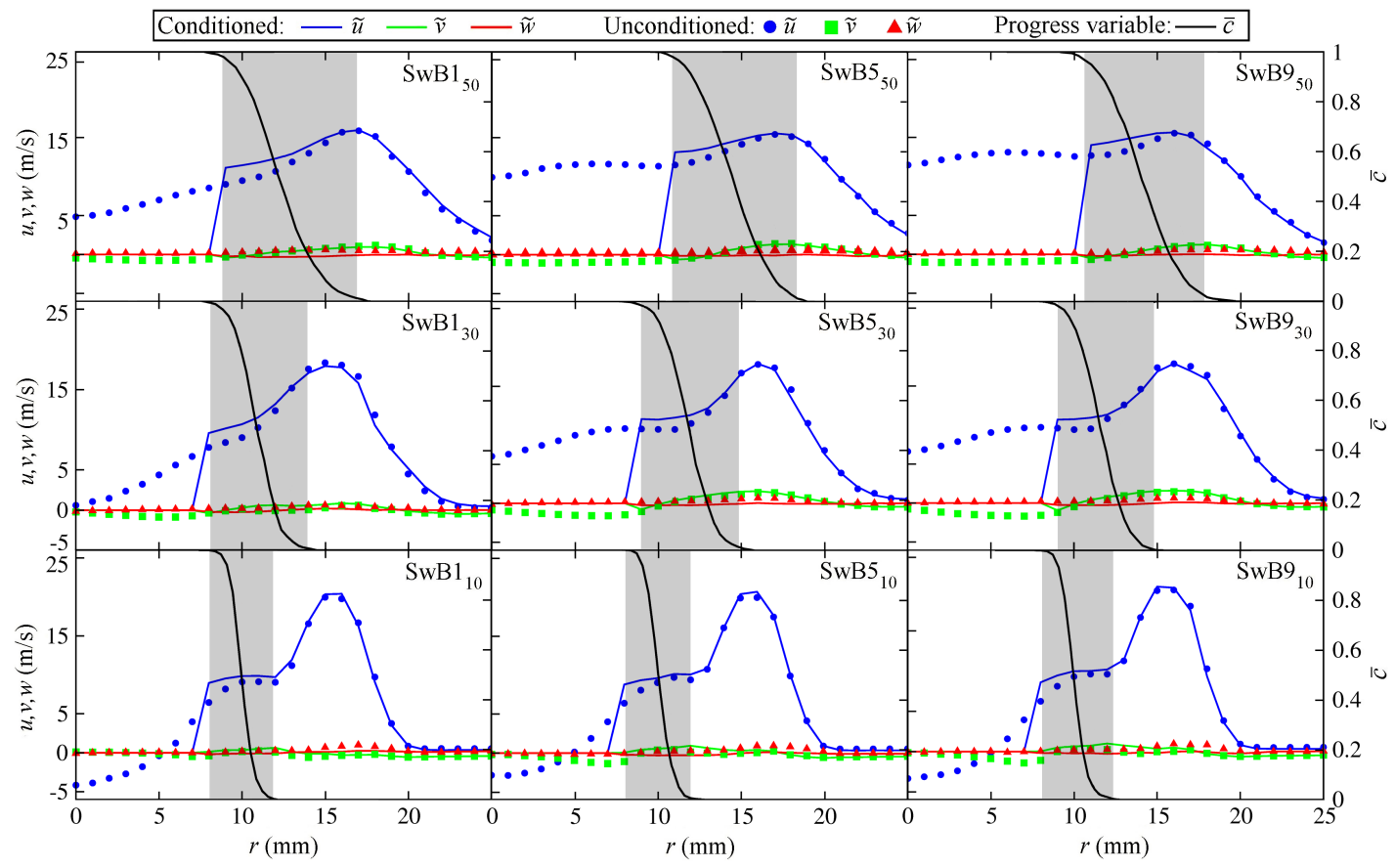

Fig. 6. Radial profiles of LDA measurements of mean conditioned reactant velocities $u, v, w$ representing axial, radial, and tangential directions (solid lines), the unconditioned LDA measurements (symbols), and progress of reaction (solid black line) for three different axial positions (rows) for case SwB1 (left), SwB5 (middle) and SwB9 (right). 


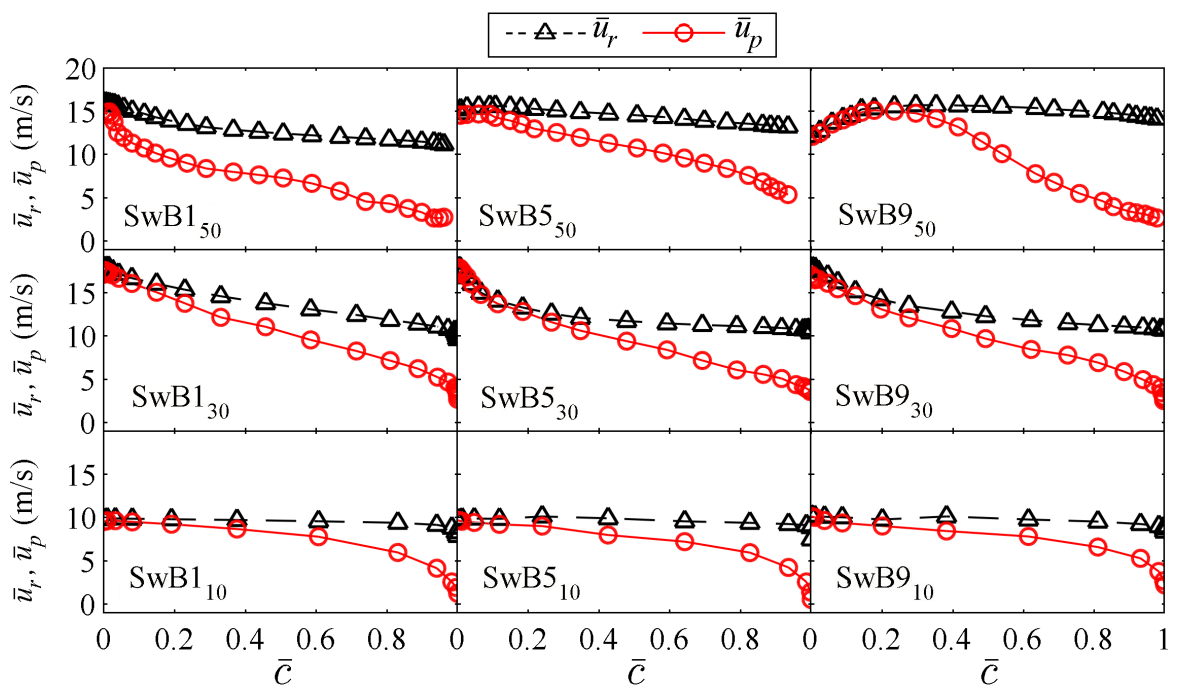

Fig. 7. Radial profiles of calculated product and measured conditioned reactant axial velocities extracted at three different axial positions (rows) in the flame brush region (rows). 


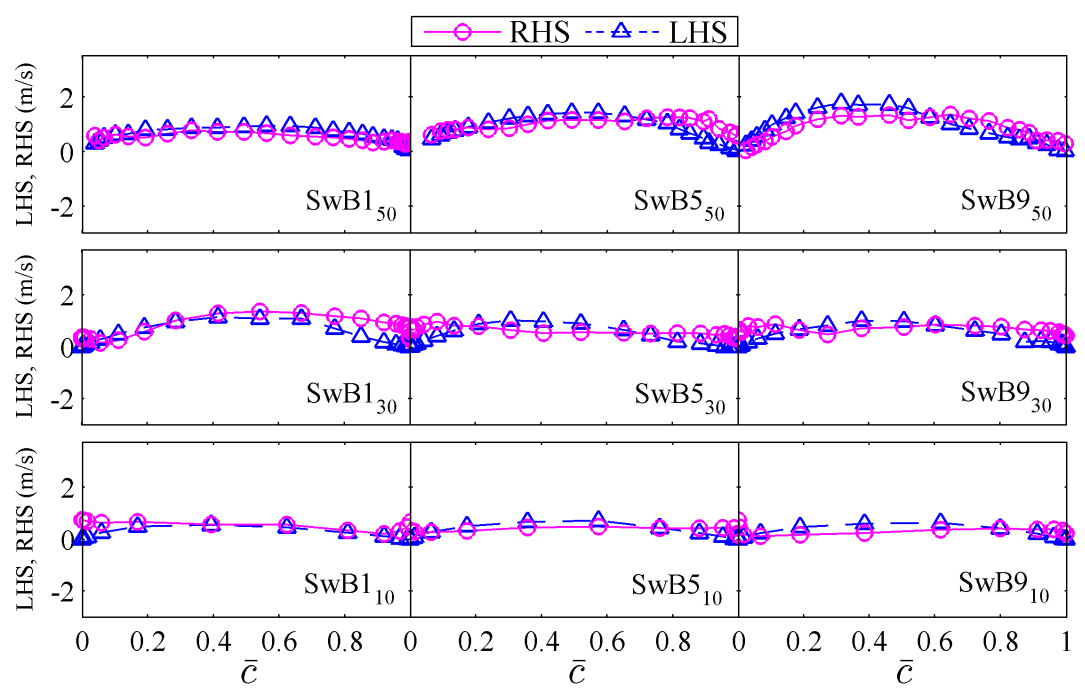

Fig. 8. LHS $=(\bar{u}-\tilde{u})$, and RHS $=(\bar{c}-\tilde{c})\left(\bar{u}_{r}-\bar{u}_{p}\right)$ from Eq. (2) as a function of progress of reaction, extracted at three different axial positions (rows) for all the three cases 